\title{
Juegos didácticos en el aula: experimentación para el aprendizaje en la asignatura de Ciencias Aplicadas I del Grado de Conservación y Restauración de Bienes Culturales
}

\section{Educational games in the classroom: experimentation for learning in the subject of Applied Sciences I of the Degree of Conservation and Restoration of Cultural Heritage}

ELENA VÁZQUEZ JIMÉNEZ

ORCID: http://orcid.org/0000-0002-7297-7633

Universidad de Sevilla

Departamento de Pintura

Evazquez5@us.es

Fecha de recepción:

Fecha de aceptación:

DOI: http://dx.doi.org/10.12795/9788447221912.126

Pp.: 2261-2286 


\section{Resumen}

Este Ciclo de Mejora en el Aula (CIMA) ha sido experimentado durante el Curso General de Docencia Universitaria. Se aplicó durante el primer cuatrimestre del curso 2019/2020 en la asignatura de Ciencias Aplicadas I. Esta materia se encuadra en el segundo curso del Grado en Conservación y Restauración en la Facultad de Bellas Artes de la Universidad de Sevilla.

La experiencia didáctica se ha basado en pasar de una práctica tradicional a un modelo didáctico distinto teniendo en cuenta los modelos mentales de los estudiantes y sus necesidades específicas. Se ha fomentado el aprendizaje reconfigurando el aula mediante experiencias en las que el docente ha dejado de ser el centro de atención y el estudiante ha adquirido el control de su aprendizaje.

Palabras clave: Ciclo de Mejora, experimentación, conservación y restauración de Bienes Culturales, juegos didácticos.

\section{Abstract}

The Second Improvement Cycle in Classroom has been experienced during the General Course of University Teaching. It was applied during the first four months of the 2019/2020 course in the subject of Applied Sciences I. This subject is part of the second course of the Degree in Conservation and Restoration at the Faculty of Fine Arts of the University of Seville.

The didactic experience has been based on moving from a traditional practice to a different didactic model taking into account the mental models of the students and their specific needs. Learning has been encouraged by reconfiguring the classroom through experiences in which the teacher has ceased to be the center of attention and the student has acquired control of their learning.

key words: Improvement Cycle in Classroom, experimentation, Conservation and Restoration of Cultural Heritage, Educational games.

Jornadas de Formación e Innovación Docente del Profesorado | № 2 (2019)

Esta obra se distribuye con la licencia Creative Commons 


\section{Descripción del contexto}

Esta asignatura es de carácter obligatorio y está inscrita en el primer cuatrimestre del Grado. Sus actividades formativas son de índole teórico-práctica y se encuentran repartidas en dos sesiones de dos horas cada una a la semana. Este curso académico cuenta con 65 alumnos matriculados, repartidos entre el grupo de mañana y tarde y de los que el $20 \%$ repiten materia.

En respuesta a la ordenación temporal de los contenidos establecidos en el Programa Docente vigente este curso, el Ciclo de Mejora ha sido diseñado y aplicado en dos de los doce temas que conforman el programa. Concretamente ha coincidido con el temario del Bloque II dedicado a "Naturaleza de los materiales de los Bienes Culturales y de Intervención": a los temas dedicados a los "Materiales inorgánicos: soportes, cargas inertes y pigmentos" y a los "Materiales orgánicos: soportes, aglutinantes, colorantes y barnices". La evaluación establecida en el programa recoge entre los aspectos a calificar, la actitud del alumno ante la asignatura, la entrega de trabajos teórico- prácticos y la superación de una prueba teórica tipo test.

En relación directa con este sistema de evaluación se ha desarrollado un modelo didáctico cíclico estructurado: se ha partido de un problema inicial y mediante el planteamiento de problemas encadenados se han desarrollado distintas actividades de contraste teniendo en cuenta la información aportada por el alumno y sus modelos mentales. El contenido final del ciclo se basa en las conclusiones resultantes de las ideas colectivas de los estudiantes.

Se ha diseñado una experiencia por tanto completa, en la que se ha partido de una pregunta inicial genérica que se ha encadenado a una secuencia subordinada de preguntas diseñadas para estimular el proceso de razonamiento del grupo. A cada tema de la asignatura se le asignó

Jornadas de Formación e Innovación Docente del Profesorado | № 2 (2019)

Esta obra se distribuye con la licencia Creative Commons 
un color ( 6 colores en total, dos por cada tema) y tanto las preguntas como las respuestas que han constituido el temario han quedado impresas en tarjetas individuales a modo de herramienta didáctica disponible para un repaso previo de la materia antes de la realización de la prueba teórica.
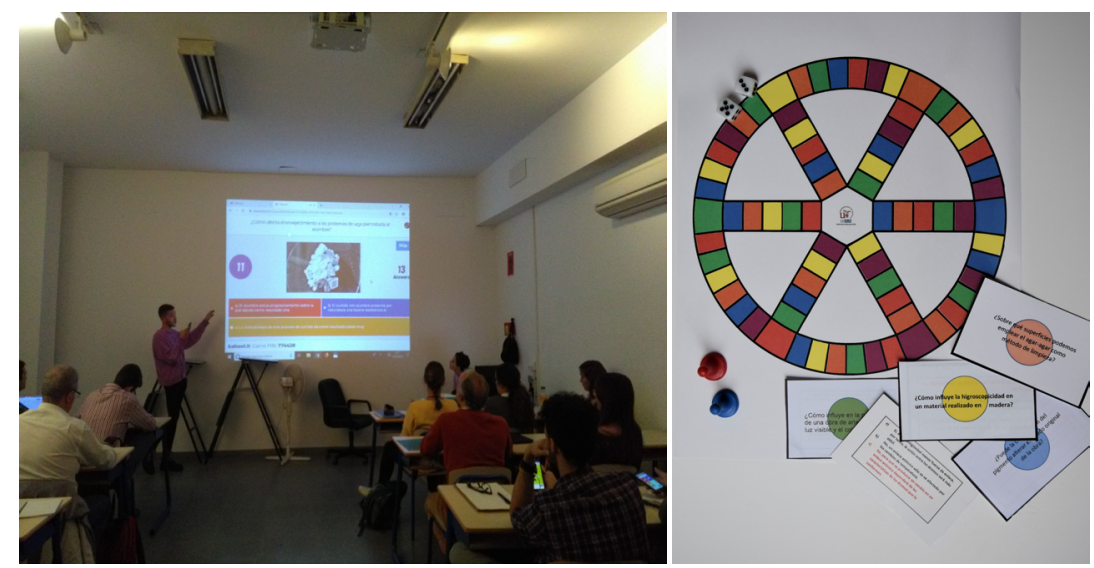

Figura 1 y Figura 2. En la imagen de la izquierda se observa uno de los alumnos haciendo uso de la aplicación Kahoot en el aula. En la imagen de la derecha prototipo del juego didáctico elaborado en el aula.

Jornadas de Formación e Innovación Docente del Profesorado | № 2 (2019)

Esta obra se distribuye con la licencia Creative Commons Reconocimiento-NoComercial-SinObraDerivada Internacional (CC BY-NC-ND 4.0.) 


\section{Diseño previo del Ciclo de Mejora Docente}

\section{Mapa de contenidos y problemas}

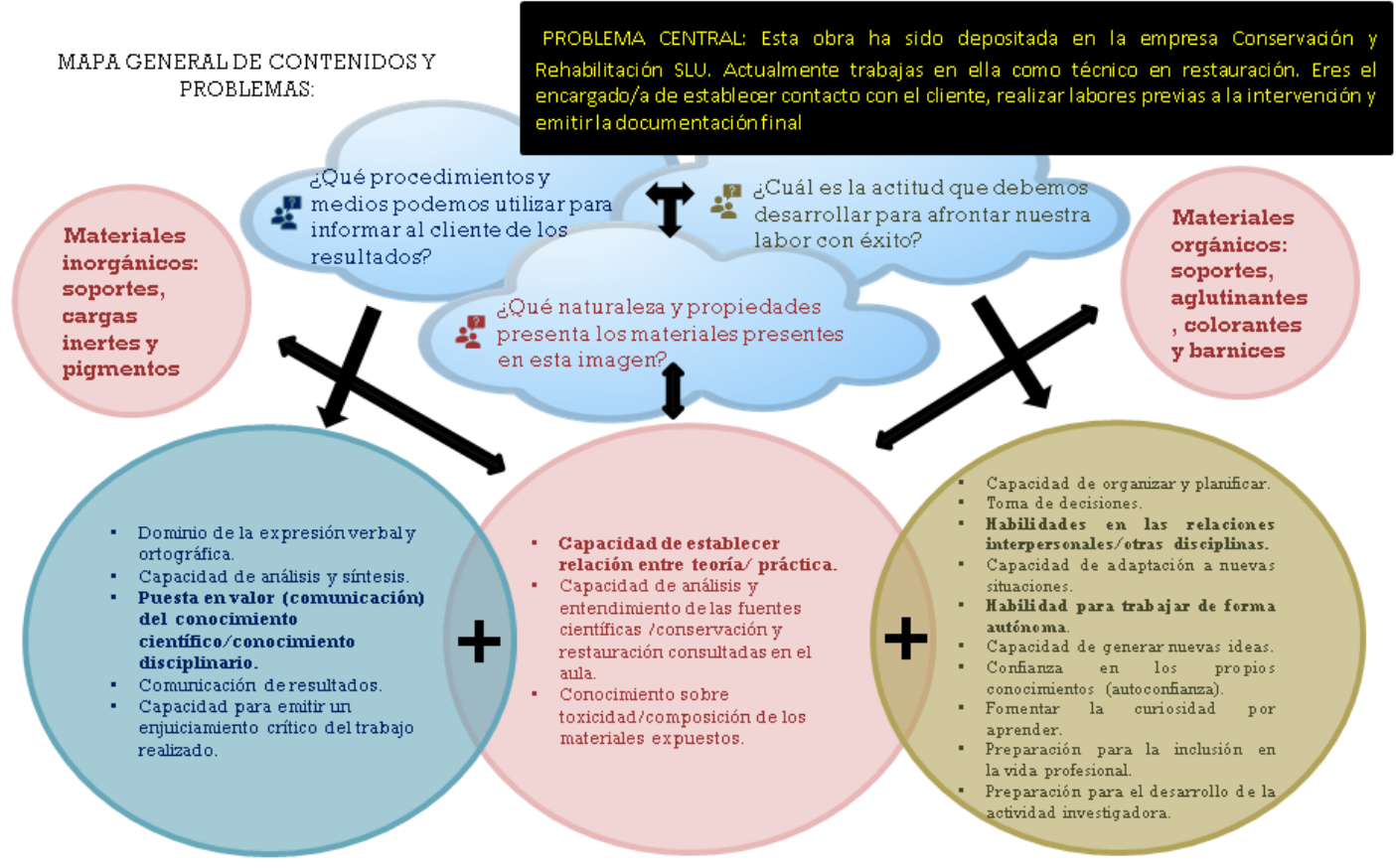

Figura 3. Mapa de contenidos y problemas.

Jornadas de Formación e Innovación Docente del Profesorado | № 2 (2019) 
En el Ciclo de Mejora se han ligado los conceptos específicos propios de la materia, determinadas habilidades y una serie de actitudes que forman parte de nuestra disciplina. Los contenidos conceptuales se han estructurado en base a la necesidad de lograr establecer relaciones entre los conceptos específicos y su aplicación a la profesión. En este aspecto se han tenido en cuenta otros contenidos secundarios como el análisis y manejo de fuentes científicas y el conocimiento tanto de la naturaleza de los materiales que interactúan en una obra de arte como de los productos que se emplean en las restauraciones y la toxicidad de estos últimos.

El análisis de determinados contenidos tanto procedimentales como actitudinales trabajados durante el curso, han permitido comprobar su importancia dentro de nuestra profesión. Esta cuestión ha sido determinante tanto para introducirlos, como para dotarles de un mayor protagonismo en los temas de la asignatura. Los primeros se han estructurado en base a la necesidad de comunicar un trabajo basado en una metodología científica, aunque teniendo también en cuenta otros contenidos secundarios como el dominio de la expresión verbal y ortográfica, la capacidad de análisis y sintesis, la necesidad divulgar la labor conservadora-restauradora y la capacidad para emitir un juicio crítico del trabajo realizado. Respecto a los segundos, se ha insistido en la necesidad de desarrollar habilidades tanto en las relaciones interpersonales y con otras disciplinas como para trabajar de forma autónoma. Asimismo, se ha considerado necesario subrayar la capacidad de organizar y planificar el trabajo, la importancia de la toma de decisiones, la capacidad de adaptación a nuevas situaciones, la capacidad de generar nuevas ideas, la habilidad, la autoconfianza, y la adquisición por parte de los estudiantes de las claves necesarias para su inclusión en la vida profesional y en la actividad investigadora.

Jornadas de Formación e Innovación Docente del Profesorado | № 2 (2019)

Esta obra se distribuye con la licencia Creative Commons 


\section{Modelo metodológico posible y secuencias de actividades programadas}

En la aplicación de los contenidos propuestos en el CIMA el alumno ha jugado un papel esencial como protagonista en el aula, logrando construir sus propios significados. En el diseño del modelo metodológico se incluye una secuencia de actividades en la que interactúan los tres problemas anteriormente descritos y que se han considerado motivadores para el aprendizaje de la asignatura. Se trata de un modelo metodológico cíclico en base a cuatro actividades: dos de contraste y otras dos basadas en las aportaciones de los alumnos.

Jornadas de Formación e Innovación Docente del Profesorado | № 2 (2019) 
MODELO METOLODÓGICO PROPUESTO EN EL SE GUND O CIMA

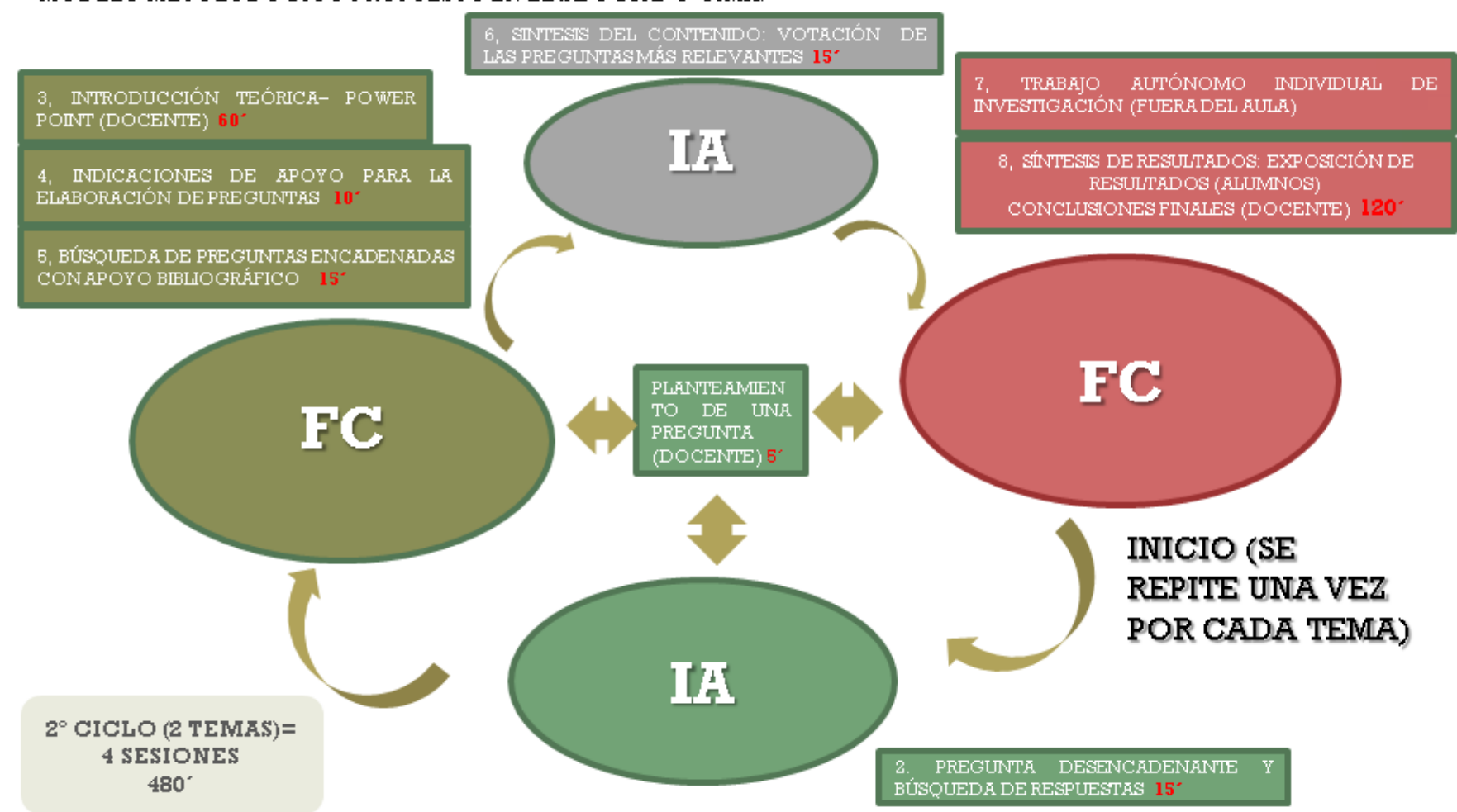

Figura 4. Modelo Metodológico posible

Jornadas de Formación e Innovación Docente del Profesorado | № 2 (2019) 
A continuación se exponen tres tablas en las que se especifican las distintas actividades y su relación con los objetivos marcados en cada bloque de contenido.

a) Primer bloque de contenidos: ¿Qué naturaleza y propiedades presenta los materiales presentes en esta imagen?

Tabla 1

\begin{tabular}{|c|c|}
\hline \multicolumn{2}{|c|}{$\begin{array}{l}\text { CONTENIDOS CONCEPTUALES: } \\
\text { ¿Qué naturaleza y propiedades presenta los materiales presentes en esta imagen? }\end{array}$} \\
\hline OBJETIVO/CONTENIDO & ACTIVIDADES EN LAS QUE SE APLICA: \\
\hline $\begin{array}{l}\text { - Conocimiento teórico } \\
\text { sobre el contenido } \\
\text { especifico del tema } 4 \text { y } 5 \\
\text { (estructural) }\end{array}$ & $\begin{array}{l}\text {-(2) Pregunta desencadenante y búsqueda de } \\
\text { respuestas (IA) } \\
\text {-(3) Introducción teórica } \\
\text {-(5) Búsqueda de preguntas encadenadas con apoyo } \\
\text { bibliográfico (FC) } \\
\text {-(7) Trabajo autónomo de investigación (FC) }\end{array}$ \\
\hline $\begin{array}{l}\text {-Capacidad de establecer } \\
\text { relación entre teoria/ } \\
\text { práctica (estructural). } \\
\text {-Conocimiento sobre } \\
\text { toxicidad/composición de } \\
\text { los materiales. }\end{array}$ & $\begin{array}{l}\text {-(3) Introducción teórica } \\
\text {-(5) Búsqueda de preguntas encadenadas con apoyo } \\
\text { bibliográfico (FC) } \\
\text {-(7) Trabajo autónomo de investigación (FC) }\end{array}$ \\
\hline $\begin{array}{l}\text { - Capacidad de análisis } \\
\text { y entendimiento de } \\
\text { las fuentes científicas } \\
\text { /conservación y } \\
\text { restauración consultadas } \\
\text { en el aula. }\end{array}$ & $\begin{array}{l}\text {-(4) Indicaciones de apoyo para la elaboración de } \\
\text { preguntas } \\
\text {-(5) Búsqueda de preguntas encadenadas con apoyo } \\
\text { bibliográfico (FC) } \\
\text {-(7) Trabajo autónomo de investigación (FC) }\end{array}$ \\
\hline \multicolumn{2}{|c|}{$\begin{array}{l}\text { Nota: el número entre paréntesis que aparece delante de cada actividad se } \\
\text { corresponde con la numeración que recibe dicha actividad en la representación } \\
\text { del Modelo Metodológico Posible. Los objetivos estructurales se han marcado en } \\
\text { negrilla (Figura 2). }\end{array}$} \\
\hline
\end{tabular}

De los dos objetivos estructurales planteados en este punto del Ciclo de Mejora uno de ellos es la adquisición de Conocimiento teórico sobre el temario. Para ello, la sesión se inicia planteando un problema sugerente a fin de estimular la curiosidad de los estudiantes y activar su razonamiento. En este punto, las ideas iniciales aportadas por

Jornadas de Formación e Innovación Docente del Profesorado | № 2 (2019)

Esta obra se distribuye con la licencia Creative Commons Reconocimiento-NoComercial-SinObraDerivada $\quad 4.0$ Internacional (CC BY-NC-ND 4.0.) 
los alumnos suponen una herramienta de gran utilidad a la hora de conectar sus modelos mentales con los conceptos "teóricos" que conforman el tema.

A continuación, se desarrollan una serie de actividades de contraste: una introducción al tema (en PowerPoint) en la que se expone el marco teórico y se debate sobre los conceptos básicos incluidos en el programa. A partir del análisis de distintos artículos científicos en el aula los estudiantes formulan preguntas desencadenantes. La sintesis de los contenidos expuestos durante el transcurso de la sesión se realiza al seleccionar entre todos las preguntas planteadas de mayor interés. La búsqueda de las respuestas se convierte en un Trabajo de Investigación desarrollado por un grupo de alumnos asignados a cada tema. Este trabajo autónomo se lleva a cabo fuera de clase y supone una herramienta de aprendizaje mediante la que el estudiante llega a sus propias conclusiones de manera crítica y razonada. Por tanto, el ciclo queda cerrado con otra actividad de contraste en la que los alumnos exponen y sintetizan los contenidos teóricos a los que han llegado en el desarrollo de sus trabajos de investigación.

El otro objetivo consiste en establecer relaciones entre la teoría y la vida laboral. Con este fin se introduce durante la exposición ejemplos de casos reales (ej: intervenciones o investigaciones en las que se han empleado o investigado sobre esos materiales). Tanto para la elaboración de las preguntas en el aula como en el momento del desarrollo del trabajo autónomo, los alumnos cuentan con diversos artículos científicos de interés que ellos buscan o se les proporcionan y mediante los que también afianzan esta relación. Estas tres actividades además les ayudan alcanzar otros objetivos como es el desarrollo de un conocimiento específico sobre el comportamiento, naturaleza y toxicidad de los materiales. Asimismo, se considera importante desarrollar la capacidad de análisis de las fuentes científicas para ejercer esta profesión y entrar

Jornadas de Formación e Innovación Docente del Profesorado | № 2 (2019)

Esta obra se distribuye con la licencia Creative Commons 
en una dinámica de continuo reciclaje y actualización. Por ello el manejo común de las fuentes bibliográficas y la selección de la información suponen dos actividades básicas transversales.

b) Segundo bloque de contenidos: ¿Qué procedimientos y medios podemos utilizar para informar al cliente de los resultados?

Tabla 2

\begin{tabular}{|c|c|}
\hline \multicolumn{2}{|c|}{$\begin{array}{l}\text { CONTENIDOS PROCEDIMENTALES: } \\
\text { ¿Qué procedimientos y medios podemos utilizar para informar al cliente de los } \\
\text { resultados? }\end{array}$} \\
\hline OBJETIVO/CONTENIDO & ACTIVIDADES EN LAS QUE SE APLICA: \\
\hline $\begin{array}{l}\text {-Dominio de la expresión } \\
\text { verbal y ortográfica. } \\
\text {-Capacidad de análisis y } \\
\text { síntesis. }\end{array}$ & $\begin{array}{l}\text {-(2) Pregunta desencadenante y búsqueda de } \\
\text { respuestas (IA). } \\
\text {-(4) Indicaciones de apoyo para la elaboración de } \\
\text { preguntas } \\
\text {-(5) Búsqueda de preguntas encadenadas con } \\
\text { apoyo bibliográfico (FC) } \\
\text {-(7) Trabajo autónomo de investigación (FC) }\end{array}$ \\
\hline $\begin{array}{l}\text {-Puesta en valor } \\
\text { (comunicación) del } \\
\text { conocimiento científico/ } \\
\text { conocimiento disciplinario } \\
\text { (estructural). } \\
\text {-Comunicación de resultados. }\end{array}$ & $\begin{array}{l}\text {-(6) Sintesis de contenido: votación de las } \\
\text { preguntas más relevantes (IA) } \\
\text {-(8) Exposición de resultados (alumno) (FC) }\end{array}$ \\
\hline $\begin{array}{l}\text {-Capacidad para emitir un } \\
\text { enjuiciamiento crítico del } \\
\text { trabajo realizado. }\end{array}$ & $\begin{array}{l}\text {-(6) Sintesis de contenido: votación de las } \\
\text { preguntas más relevantes (IA) } \\
\text {-(7) Trabajo autónomo de investigación (FC) } \\
\text {-(8) Síntesis de resultados: Exposición de } \\
\text { resultados (alumnos) y conclusiones finales } \\
\text { (docente)(FC) }\end{array}$ \\
\hline \multicolumn{2}{|c|}{$\begin{array}{l}\text { Nota: el número entre paréntesis que aparece delante de cada actividad se } \\
\text { corresponde con la numeración que recibe dicha actividad en la representación } \\
\text { del Modelo Metodológico Posible. Los objetivos estructurales se han marcado en } \\
\text { negrilla (Figura 2). }\end{array}$} \\
\hline
\end{tabular}

En este bloque se han marcado también distintos objetivos. Dos de ellos consisten en estimular el dominio de la expresión verbal y ortográfica además de la capacidad de análisis y sintesis. Para ello se propusieron distintas

Jornadas de Formación e Innovación Docente del Profesorado | № 2 (2019)

Esta obra se distribuye con la licencia Creative Commons Reconocimiento-NoComercial-SinObraDerivada $\quad 4.0$ Internacional (CC BY-NC-ND 4.0.) 
actividades que suponen un esfuerzo para el estudiante en el uso del lenguaje científico tanto escrito como oral. Estos dos objetivos se estimulan mediante la realización del Cuaderno de Actividades, que recoge la pregunta inicial y su razonamiento, y el Trabajo de Investigación que sintetiza la investigación realizada, además de ser expuesto en el aula y entregado por escrito.

Dos objetivos estructurales dentro de este bloque son la puesta en valor del conocimiento disciplinario adquirido y la comunicación de los resultados obtenidos. Con la intención de despertar interés por la materia y estimular la curiosidad del alumnado a la hora de poner en valor el conocimiento, las actividades se enfocaron en torno al estudio de la materia que suscitó mayor curiosidad. La comunicación de los resultados es una herramienta que ha servido de ayuda para que expresen sus ideas, y constituye un objetivo presente tanto en la exposición oral ante los compañeros como en el Trabajo de Investigación, que es subido a la enseñanza virtual y compartido por el resto de los compañeros, pasando a formar parte del temario de la prueba teórica.

El enjuiciamiento crítico del trabajo realizado se encuentra también latente en estas actividades. Mediante la votación del temario expresan el interés que puede llegar a suscitar sus preguntas frente a las demás. Asimismo, mediante el Trabajo de Investigación ahondan en la materia y persiguen respuestas que posteriormente defienden de manera crítica ante sus compañeros.

c) Tercer bloque de contenidos: ¿Cuál es la actitud que debemos desarrollar para afrontar nuestra labor con éxito?

Jornadas de Formación e Innovación Docente del Profesorado | № 2 (2019)

Esta obra se distribuye con la licencia Creative Commons 
Tabla 3

CONTENIDOS ACTITUDINALES:

¿Cuál es la actitud que debemos desarrollar para afrontar nuestra labor con éxito?

\begin{tabular}{|l|l|}
\hline OBJETIVO/CONTENIDO & ACTIVIDADES EN LAS QUE SE APLICA: \\
\hline -Capacidad de organizar y & -(2) Pregunta desencadenante y búsqueda de \\
planificar. & respuestas (IA) \\
-Toma de decisiones. & $-(3)$ Introducción teórica \\
-Habilidades en las relaciones & $-(4)$ Indicaciones de apoyo para la elaboración de \\
interpersonales/otras & preguntas.
\end{tabular}

disciplinas (estructural).

- Capacidad de adaptación a

nuevas situaciones.

-Habilidad para trabajar de

forma autónoma (estructural).

-Capacidad de generar nuevas

ideas.

- Confianza en los propios

conocimientos (autoconfianza).

- Fomentar la curiosidad por

aprender.

-Preparación para la inclusión

en la vida profesional.

- Preparación para el

desarrollo de la actividad

investigadora.

Nota: el número entre paréntesis que aparece delante de cada actividad se corresponde con la numeración que recibe dicha actividad en la representación del Modelo Metodológico Posible. Los objetivos estructurales se han marcado en negrilla (Figura 2).

Los contenidos actitudinales forman parte de todas las actividades desarrolladas en este ciclo. En esta disciplina, estos objetivos constituyen una parte esencial del trabajo diario; los conservadores-restauradores se mueven habitualmente en ambientes de trabajo con otras disciplinas y con instituciones de diversa índole. En muchas ocasiones se realizan intervenciones en grupo y en otras se trabaja de forma totalmente autónoma. En este último caso es necesario desarrollar capacidades para organizar y planificar el trabajo a la vez que para tomar continuas decisiones y generar nuevas ideas.

Jornadas de Formación e Innovación Docente del Profesorado I № 2 (2019)

Esta obra se distribuye con la licencia Creative Commons Reconocimiento-NoComercial-SinObraDerivada $\quad 4.0$ Internacional (CC BY-NC-ND 4.0.) 


\section{Cuestionario Inicial-final}

Con el fin de analizar los modelos de los estudiantes se diseñó un cuestionario Inicial-final que permitiera registrar su evolución en relación con los problemas planteados en el aula. Los cuestionarios fueron repartidos tanto en el grupo de mañana como en el de tarde. Aunque fueron anónimos, el alumnado identificó su cuestionario inicial mediante un seudónimo para poder localizarlo a la hora de realizar el cuestionario final. De los 65 alumnos matriculados en la asignatura se recogieron 43 encuestas iniciales (23 del grupo de mañana y 20 del grupo de tarde), quedando 8 de ellas incompletas, y por tanto no fueron incluidas en el cuestionario final. Teniendo en cuenta estos datos, la participación del alumnado en este instrumento de seguimiento del estudiante fue del 54,6\%.

Para facilitar a los alumnos la tarea se proyectó una imagen en el aula con la finalidad de conectarlos con la vida laboral real. Se les planteó el siguiente problema: Esta obra ha sido depositada en la empresa Conservación y Rehabilitación SLU. Actualmente trabajas en ella como técnico en restauración. Eres el encargado/a de establecer contacto con el cliente, realizar labores previas a la intervención y emitir la documentación final.

En torno a este problema se diseñaron tres cuestiones:

1. Indica el tipo, naturaleza y propiedades de los distintos materiales que constituyen este bien.

2. Indica con el máximo de detalle todos los procedimientos y los medios que tenemos que utilizar para informar al cliente de los resultados de la restauración.

3. Describe todas las actitudes que debemos desarrollar para afrontar nuestra labor con éxito.

Jornadas de Formación e Innovación Docente del Profesorado | № 2 (2019) 


\section{Aplicación del Ciclo de Mejora Docente}

\section{Relato resumido de las sesiones}

Este apartado se resume en dos cuestiones claves;

¿Qué ha funcionado en el CIMA? El diseño de las actividades y el tiempo dedicado a cada una de ellas ha funcionado bastante bien. Aunque en un principio hubo pequeños imprevistos, el resultado ha sido bastante satisfactorio y finalmente se pudieron aplicar todas las actividades previstas. Este método activo ha obligado a los alumnos a sentirse cada vez más involucrados en la asignatura. Además, en el transcurso de la experiencia, cada vez se han encontrado más cómodos y han mostrado paulatinamente mayor interés. Aunque a un cierto número de alumnos les costó asimilar este sistema de trabajo, con el avance de la asignatura todos han ido tomando este sistema cada vez más como el habitual.

La implicación de los alumnos creció notablemente a lo largo del desarrollo de la experiencia. Aunque probablemente no todos terminaron completamente convencidos de la eficacia de este sistema, algunos han manifestado que se encuentran satisfechos al poder establecer conexión entre la teoría expuesta en clase y las labores de conservación y restauración que van a desarrollar en el futuro. El compromiso es un aspecto que no ha funcionado al mismo nivel en los dos grupos; mientras que en uno de ellos ha sido la tónica general en el otro es un aspecto para trabajar más profundamente.

Jornadas de Formación e Innovación Docente del Profesorado | № 2 (2019) 
¿Qué mejoras se podrían introducir en el CIMA planteado?

Es necesario seguir ajustando los procedimientos y métodos de trabajo y establecer muy bien las pautas e instrucciones. Muchos de los alumnos se encontraron con dificultad para relacionar la teoría con los artículos trabajados en el aula. Señalarles palabras claves en los artículos e incluso preguntas genéricas facilitó en gran medida el desarrollo y entendimiento esta actividad de contraste. Con el avance de la asignatura cada vez se han ido facilitando los artículos menos trabajados, esta medida no ha ido en detrimento de la calidad de las preguntas, las cuales han sido formuladas cada vez mejor. Con el trabajo autónomo ha ocurrido una experiencia similar, enviar a los alumnos enlaces de interés para desarrollar las respuestas a las preguntas seleccionadas se ha visto reflejado en un resultado que ha ido mejorando notablemente.

La experiencia desarrollada en el trascurso de la asignatura ha ayudado a concluir que la elección del material (en este caso artículos de carácter científico) supone un punto importante para los resultados obtenidos.

\section{Evaluación del aprendizaje de los estudiantes. Escaleras de Aprendizaje.}

Las escaleras de aprendizaje han supuesto una herramienta de gran utilidad para representar la progresión de aprendizaje de los alumnos en distintos niveles y así poder detectar los posibles obstáculos.

Jornadas de Formación e Innovación Docente del Profesorado | № 2 (2019)

Esta obra se distribuye con la licencia Creative Commons 


\section{ELENA VÁZQUEZ JIMÉNEZ}

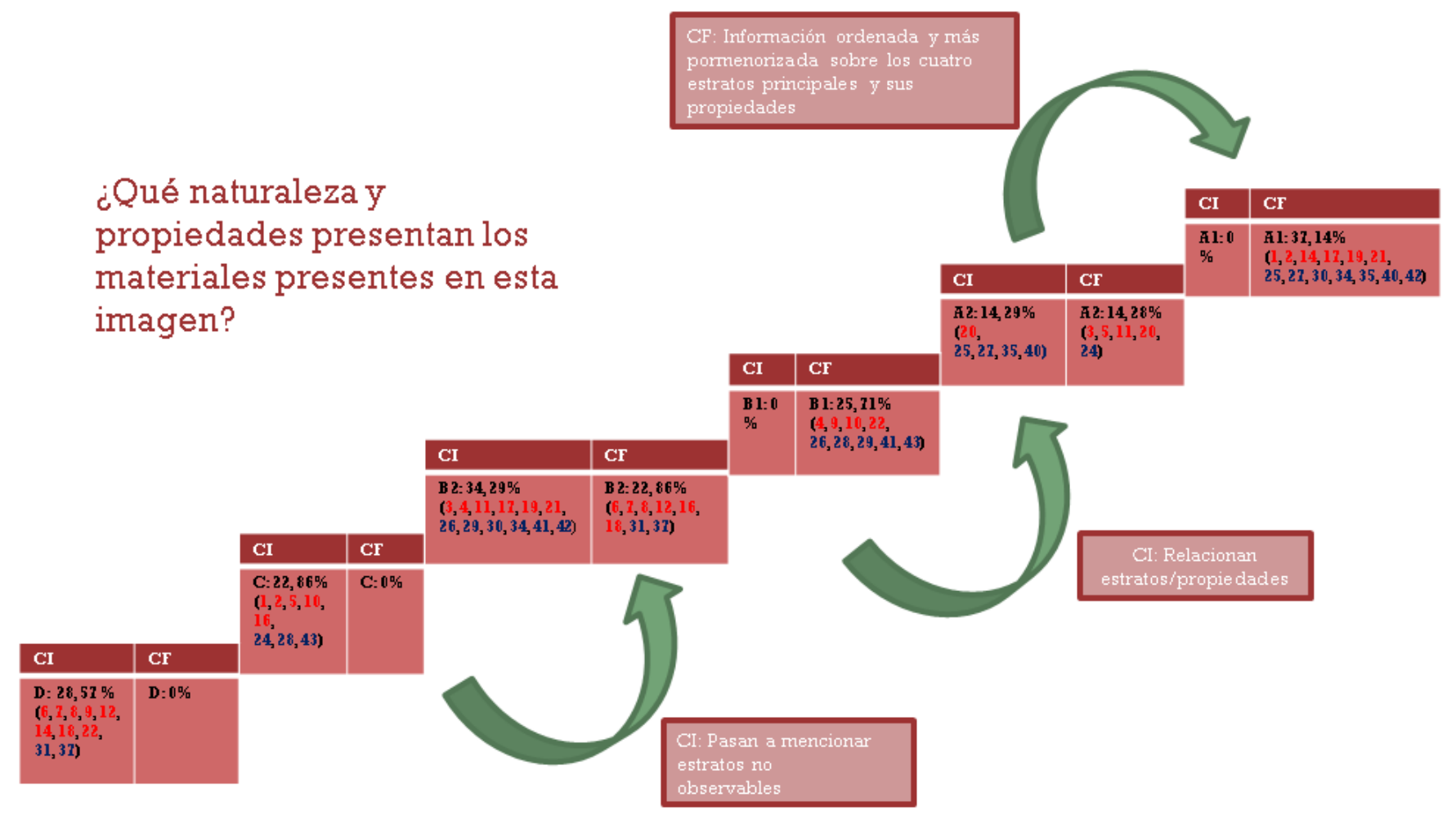

Figura 5. Escalera de aprendizaje correspondiente a la pregunta 1. En estas tres escaleras la numeración roja se corresponde con los cuestionarios del grupo de la mañana y la azul con los del grupo de tarde. Esta numeración permite observar el avance individualizado de cada alumno.

Jornadas de Formación e Innovación Docente del Profesorado | № 2 (2019) 
¿Qué procedimientos y medios podemos utilizar para informar al cliente de los resultados?

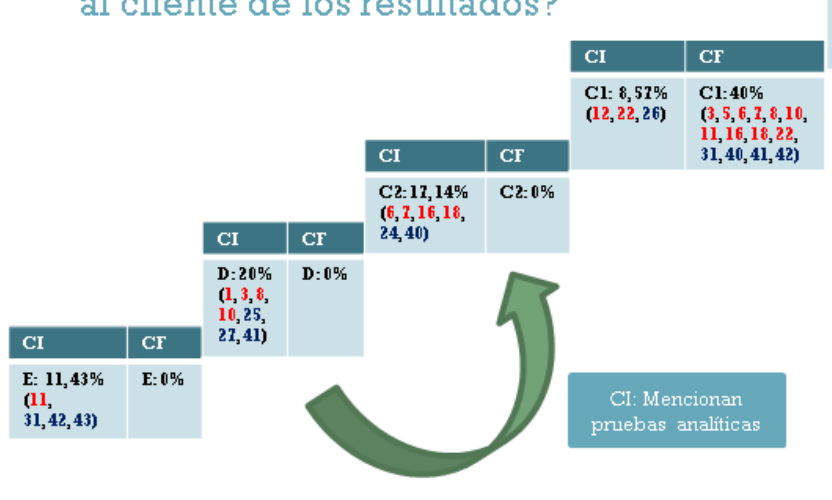

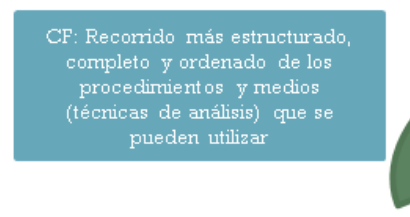

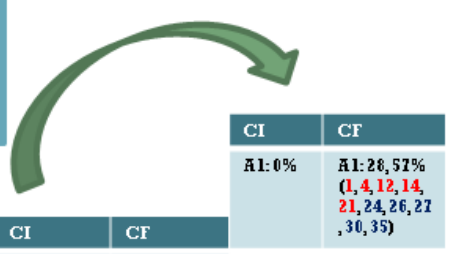

A2:14, 28\% $\quad$ A2:25, $11 \%$

$\begin{array}{ll}(4,14, & (2,9,17,19, \\ 21,30,35) & 25,28,34,\end{array}$
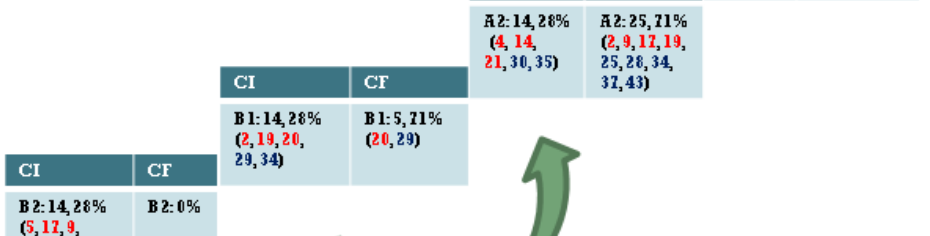

B 1: $5,71 \%$

B 2: 14, 28

$(5,17,9$,
$28,37)$

B 2:0\%

$(2,19,20$,

$(20,29)$

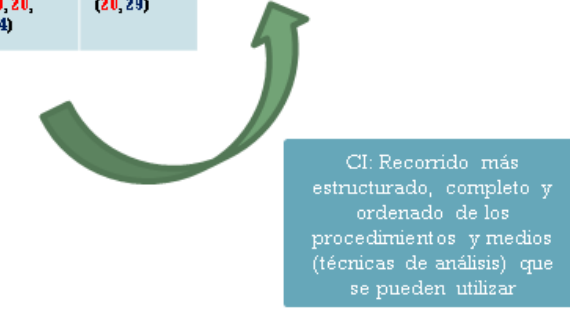

Figura 6. Escalera de aprendizaje correspondiente a la pregunta 2

Jornadas de Formación e Innovación Docente del Profesorado | № 2 (2019) 
¿Cuál es la actitud que debemos desarrollar para afrontar nuestra labor con éxito?
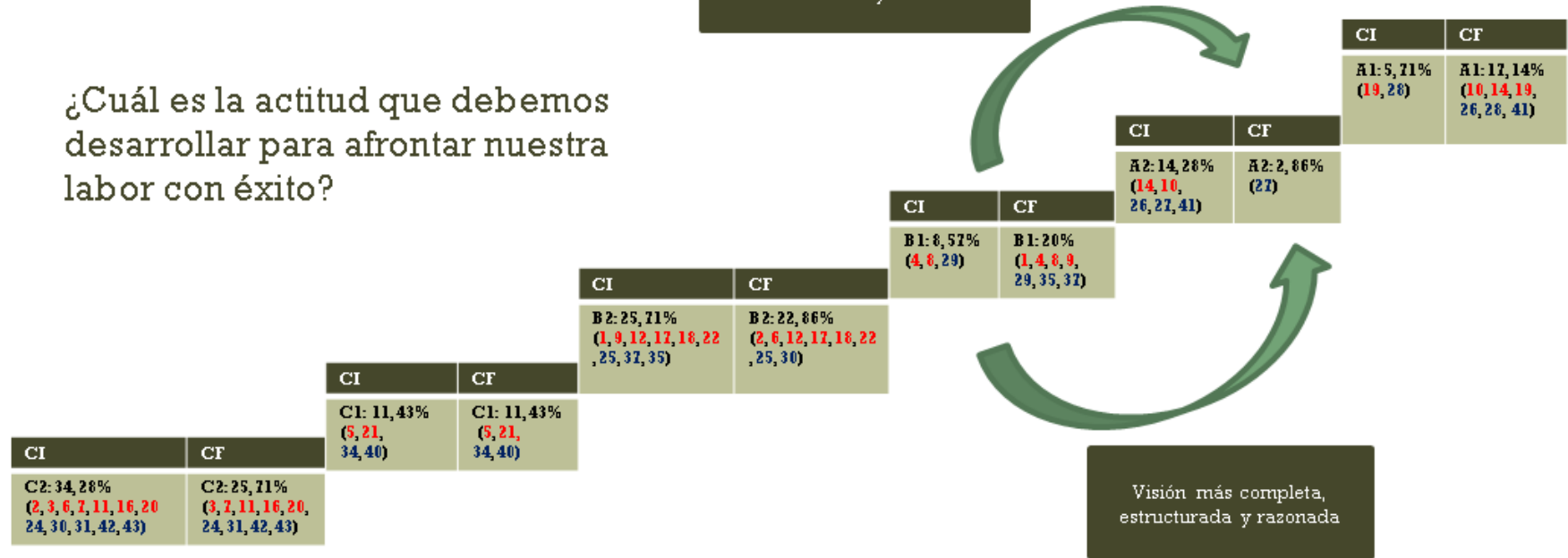

Figura 7. Escalera de aprendizaje correspondiente a la pregunta 3

Jornadas de Formación e Innovación Docente del Profesorado | № 2 (2019) 
También se elaboró una tabla que ha permitido evaluar el avance individual de cada alumno.

Tabla 4

\begin{tabular}{|c|c|c|c|c|c|c|c|c|c|}
\hline \multicolumn{10}{|c|}{ NIVELES DE DESARROLLO DE IDEAS INICIALES Y FINALES (grupo de mañana) } \\
\hline \multirow[t]{2}{*}{ № } & \multicolumn{3}{|c|}{ CUESTIÓN 1} & \multicolumn{3}{|c|}{ CUESTIÓN 2} & \multicolumn{3}{|c|}{ CUESTIÓN 3} \\
\hline & INC. & FIN. & EVOL. & INC. & FIN. & EVOL. & INC. & FIN. & EVOL. \\
\hline 1 & C & A1 & + & $\mathrm{D}$ & A1 & + & B2 & B1 & + \\
\hline 2 & C & $\mathrm{A} 1$ & + & B1 & $\mathrm{A} 2$ & + & C2 & B2 & + \\
\hline 3 & B2 & A2 & + & $\mathrm{D}$ & $\mathrm{C} 1$ & + & $\mathrm{C} 2$ & C2 & $=$ \\
\hline 4 & B2 & B1 & + & $\mathrm{A} 2$ & $\mathrm{~A} 1$ & + & B1 & B1 & $=$ \\
\hline 5 & $C$ & $\mathrm{~A} 2$ & + & B2 & $\mathrm{C} 1$ & + & C1 & C1 & $=$ \\
\hline 6 & $D$ & B2 & + & $\mathrm{C} 2$ & $\mathrm{C}_{1}$ & + & $\mathrm{C} 2$ & B2 & + \\
\hline 7 & $\mathrm{D}$ & B2 & + & $\mathrm{C} 2$ & $\mathrm{C} 1$ & + & $\mathrm{C} 2$ & C2 & $=$ \\
\hline 8 & $\mathrm{D}$ & B2 & + & $\mathrm{D}$ & $\mathrm{C} 1$ & + & B1 & B1 & $=$ \\
\hline 9 & $\mathrm{D}$ & B1 & + & B2 & $\mathrm{A} 2$ & + & B2 & B1 & + \\
\hline 10 & C & B1 & + & $\mathrm{D}$ & $\mathrm{C} 1$ & + & $\mathrm{A} 2$ & A1 & + \\
\hline 11 & B2 & $\mathrm{A} 2$ & + & $E$ & $\mathrm{C} 1$ & + & $\mathrm{C} 2$ & C2 & $=$ \\
\hline 12 & $\mathrm{D}$ & B2 & + & $\mathrm{C} 1$ & $\mathrm{~A} 1$ & + & B2 & B2 & $=$ \\
\hline 14 & $\mathrm{D}$ & A1 & + & $\mathrm{A} 2$ & $\mathrm{~A} 1$ & + & $\mathrm{A} 2$ & A1 & + \\
\hline 16 & C & B2 & + & $\mathrm{C} 2$ & $\mathrm{C} 1$ & + & C2 & $\mathrm{C} 2$ & $=$ \\
\hline 17 & B2 & A1 & + & B2 & $\mathrm{A} 2$ & + & B2 & B2 & $=$ \\
\hline 18 & D & B2 & + & $\mathrm{C} 2$ & $\mathrm{C} 1$ & + & B2 & B2 & $=$ \\
\hline 19 & B2 & A1 & + & B1 & $\mathrm{A} 2$ & + & A1 & $\mathrm{A} 1$ & $=$ \\
\hline 20 & $\mathrm{~A} 2$ & $\mathrm{~A} 2$ & $=$ & B1 & B1 & $=$ & $\mathrm{C} 2$ & $\mathrm{C} 2$ & $=$ \\
\hline 21 & B2 & A1 & + & $\mathrm{A} 2$ & $\mathrm{~A} 1$ & + & C1 & $\mathrm{C} 1$ & $=$ \\
\hline 22 & $\mathrm{D}$ & B1 & + & $\mathrm{C} 1$ & $\mathrm{C} 1$ & $=$ & B2 & B2 & $=$ \\
\hline
\end{tabular}

Tabla 5

\begin{tabular}{|c|c|c|c|c|c|c|c|c|c|}
\hline \multicolumn{10}{|c|}{ NIVELES DE DESARROLLO DE IDEAS INICIALES Y FINALES (grupo de tarde) } \\
\hline \multirow[t]{2}{*}{ No음 } & \multicolumn{3}{|c|}{ CUESTIÓN 1} & \multicolumn{3}{|c|}{ CUESTIÓN 2} & \multicolumn{3}{|c|}{ CUESTIÓN 3} \\
\hline & INC. & FIN. & EVOL. & INC. & FIN. & EVOL. & INC. & FIN. & EVOL. \\
\hline 24 & C & A2 & + & $\mathrm{C} 2$ & A1 & + & $\mathrm{C} 2$ & $\mathrm{C} 2$ & $=$ \\
\hline
\end{tabular}

Jornadas de Formación e Innovación Docente del Profesorado | № 2 (2019)

Esta obra se distribuye con la licencia Creative Commons Reconocimiento-NoComercial-SinObraDerivada Internacional (CC BY-NC-ND 4.0.) 


\begin{tabular}{|l|l|l|l|l|l|l|l|l|l|}
\hline 25 & $\mathrm{~A} 2$ & $\mathrm{~A} 1$ & + & $\mathrm{D}$ & $\mathrm{A} 2$ & + & $\mathrm{B} 2$ & $\mathrm{~B} 2$ & $=$ \\
\hline 26 & $\mathrm{~B} 2$ & $\mathrm{~B} 1$ & + & $\mathrm{C} 1$ & $\mathrm{~A} 2$ & + & $\mathrm{A} 2$ & $\mathrm{~A} 1$ & + \\
\hline 27 & $\mathrm{~A} 2$ & $\mathrm{~A} 1$ & + & $\mathrm{D}$ & $\mathrm{A} 1$ & + & $\mathrm{A} 2$ & $\mathrm{~A} 2$ & $=$ \\
\hline 28 & $\mathrm{C}$ & $\mathrm{B} 1$ & + & $\mathrm{B} 2$ & $\mathrm{~A} 2$ & + & $\mathrm{A} 1$ & $\mathrm{~A} 1$ & $=$ \\
\hline 29 & $\mathrm{~B} 2$ & $\mathrm{~B} 1$ & + & $\mathrm{B} 1$ & $\mathrm{~B} 1$ & $=$ & $\mathrm{B} 1$ & $\mathrm{~B} 1$ & $=$ \\
\hline 30 & $\mathrm{~B} 2$ & $\mathrm{~A} 1$ & + & $\mathrm{A} 2$ & $\mathrm{~A} 1$ & + & $\mathrm{C} 2$ & $\mathrm{~B} 2$ & + \\
\hline 31 & $\mathrm{D}$ & $\mathrm{B} 2$ & + & $\mathrm{E}$ & $\mathrm{C} 1$ & + & $\mathrm{C} 2$ & $\mathrm{C} 2$ & $=$ \\
\hline 34 & $\mathrm{~B} 2$ & $\mathrm{~A} 1$ & + & $\mathrm{B} 1$ & $\mathrm{~A} 2$ & + & $\mathrm{C} 1$ & $\mathrm{C} 1$ & $=$ \\
\hline 35 & $\mathrm{~A} 2$ & $\mathrm{~A} 1$ & + & $\mathrm{A} 2$ & $\mathrm{~A} 1$ & + & $\mathrm{B} 2$ & $\mathrm{~B} 1$ & + \\
\hline 37 & $\mathrm{D}$ & $\mathrm{B} 2$ & + & $\mathrm{B} 2$ & $\mathrm{~A} 2$ & + & $\mathrm{B} 2$ & $\mathrm{~B} 1$ & + \\
\hline 40 & $\mathrm{~A} 2$ & $\mathrm{~A} 1$ & + & $\mathrm{C} 2$ & $\mathrm{C} 1$ & + & $\mathrm{C} 1$ & $\mathrm{C} 1$ & $=$ \\
\hline 41 & $\mathrm{~B} 2$ & $\mathrm{~B} 1$ & + & $\mathrm{D}$ & $\mathrm{C} 1$ & + & $\mathrm{A} 2$ & $\mathrm{~A} 1$ & + \\
\hline 42 & $\mathrm{~B} 2$ & $\mathrm{~A} 1$ & + & $\mathrm{E}$ & $\mathrm{C} 1$ & + & $\mathrm{C} 2$ & $\mathrm{C} 2$ & $=$ \\
\hline 43 & $\mathrm{C}$ & $\mathrm{B} 1$ & + & $\mathrm{E}$ & $\mathrm{A} 2$ & + & $\mathrm{C} 2$ & $\mathrm{C} 2$ & $=$ \\
\hline $\begin{array}{l}\text { Nota: se han } \\
\text { incompletos. }\end{array}$
\end{tabular}

Esta tabla permite registrar y analizar el nivel o escalón en el que se encontraba cada alumno de manera individual en cada una de las preguntas planteadas al principio y al final de la aplicación del ciclo. Los resultados son los siguientes:

- En el primer problema es donde se ha producido el mayor avance. El 97,14\% de los alumnos lo han avanzado y tan sólo un alumno ha permanecido en el mismo nivel. Por tanto, las actividades llevadas a cabo en el Ciclo de Mejora en relación con la adquisición de contenidos conceptuales se considera que han logrado su objetivo.

- En el segundo problema, los resultados obtenidos en relación con los contenidos procedimentales marcados, también han sido bastante óptimos. El $91,43 \%$ de los estudiantes ha manifestado un avance, frente al 9,57\% (un alumno de tarde y dos de la mañana) que no han logrado superar los obstáculos planteados. Esta tabla sirve de instrumento para realizar un seguimiento más exhaustivo de

Jornadas de Formación e Innovación Docente del Profesorado I № 2 (2019)

Esta obra se distribuye con la licencia Creative Commons 
estos tres alumnos y poder valorar sus necesidades específicas.

- El tercer problema planteado y relacionado con la actitud ante la disciplina es el aspecto que más se debe reforzar de cara al próximo Ciclo de Mejora. Respecto a los problemas propuestos en relación con este aspecto sólo se ha producido un avance en el $31,43 \%$ de los estudiantes frente al 68,57 que se ha mantenido en el mismo nivel: 14 de los 35 alumnos del grupo de la mañana y 10 de los 15 alumnos del grupo de tarde encuestados no han experimentado un avance según los resultados obtenidos.

\section{Evaluación del Ciclo de Mejora Docente}

\section{Cuestiones a mantener y cambios a introducir para un futuro y aspectos de la experiencia que se pretenden incorporar a la práctica docente habitual}

En relación con el aprendizaje de los alumnos:

- Crear un ambiente de confianza y empatía en el que cada uno de los estudiantes se sienta parte principal en el aula.

- Reconfigurar el aula adquiriendo el papel de orientadora (no de transmisora de ideas) en la búsqueda y construcción del conocimiento.

- Impartir clases dinámicas que logren despertar su interés y mantenerlo a lo largo de todo el desarrollo de la asignatura.

- Hacerles ver la importancia de las actitudes de responsabilidad, compromiso y respeto tanto hacia los compañeros, hacia los bienes que restauramos

Jornadas de Formación e Innovación Docente del Profesorado | № 2 (2019)

Esta obra se distribuye con la licencia Creative Commons 
y hacia las instituciones o clientes con los que tratamos.

- Crear unos hábitos de aprendizaje que les ayuden a encontrar soluciones fuera del aula.

En relación con los contenidos de enseñanza:

- Conectar los conceptos teóricos de cada tema con la realidad de nuestra vida laboral, además de tener presente otros contenidos como la importancia del análisis y manejo de fuentes científicas y el conocimiento tanto de la naturaleza de los materiales que intervenimos como el de los productos que empleamos en las restauraciones, y la toxicidad de estos últimos.

- Insistir en la importancia de comunicar un trabajo basado en una metodología científica, reiterar la importancia que tiene en nuestro entorno laboral del dominio de la expresión verbal y ortográfica, la capacidad de análisis y síntesis, la necesidad de divulgar nuestra labor y la capacidad para emitir un enjuiciamiento crítico del trabajo realizado.

- Ahondar en la necesidad de desarrollar habilidades tanto para trabajar en grupo como con otras disciplinas. También se pretende insistir en otros aspectos como la importancia de la toma de decisiones, la capacidad de adaptación a nuevas situaciones, la capacidad de generar nuevas ideas, entre otros aspectos.

En cuanto a la metodología:

- Organizar secuencia de actividades en torno a una serie de problemas motivadores para el aprendizaje.

- Plantear al inicio de cada tema una pregunta o problema que permita analizar las ideas previas de los alumnos.

Jornadas de Formación e Innovación Docente del Profesorado I № 2 (2019)

Esta obra se distribuye con la licencia Creative Commons 
- Enlazar los objetivos conceptuales marcados por la asignatura con actividades de contraste para que conecten esta "teoría" con el día a día de nuestra profesión.

- Motivarlos con preguntas desencadenantes con las que estimular la construcción de su propio conocimiento.

- Realizar una estudiada síntesis de los contenidos para completar el ciclo.

En cuanto a la evaluación:

- Instrumentos de evaluación del alumnado como el cuestionario inicia, que ha resultado ser una herramienta de gran ayuda y ha permitido realizar un análisis sistemático de cada uno de los estudiantes, precisando qué saben y qué aprenden. El análisis de las respuestas permite concretar e incluso adaptar la secuencia de actividades y el mapa de contenidos, y obtener a su vez información sobre el proceso de aprendizaje de los alumnos. El cuestionario final y su representación gráfica en las escaleras de aprendizaje ha resultado ser una herramienta también muy útil para visualizar y detectar los obstáculos de aprendizaje que pueden aparecer durante las clases y poder hacer un seguimiento personalizado de cada alumno.

- Instrumentos de evaluación del aprendizaje del estudiante como la lectura crítica de fuentes científcas y la realización de trabajos escritos con los que indagan en su propio conocimiento. La exposición de ideas y la adquisición de responsabilidades en el aula, que supone la participación del estudiante y nuevas exigencias para el docente, ha quedado también aplicado a mi práctica habitual.

- Para la evaluación docente ha servido de gran ayuda el diario de trabajo, los resultados obtenidos en las encuestas finales y la elaboración de las escaleras

Jornadas de Formación e Innovación Docente del Profesorado | № 2 (2019) 
de aprendizaje. La redacción del diario ha permitido analizar el modelo didáctico planteado. Representar los resultados gráficamente en las escaleras de aprendizaje iniciales y finales permite poder revisar y mejorar tanto el diseño del modelo didáctico como su estructura y contenido. Asimismo, es de gran utilidad para realizar una valoración final las encuestas de opinión del alumnado.

\section{Principios didácticos argumentados que han guiado la experiencia y deben permanecer en un futuro}

- Desarrollar para cada tema un ciclo completo de trabajo cuyos contenidos se ajusten a las características y necesidades específicas de los estudiantes.

- Diseñar los contenidos de cada tema mediante la formulación de problemas y actividades de contraste.

- El uso de recursos de evaluación del alumnado como las escaleras de aprendizaje, la entrega de trabajos y la participación del alumnado.

Jornadas de Formación e Innovación Docente del Profesorado | № 2 (2019)

Esta obra se distribuye con la licencia Creative Commons 


\section{Referencias bibliográficas}

Finkel, D. (2008). Dar clase con la boca cerrada. Traducción Óscar Barberá. Valencia: Universidad de Valencia.

Porlán, R. (coord.) et al (2017). Enseñanza universitaria. Cómo mejorarla. Madrid: Ediciones Morata, SL.

Bain, K. (2007). Lo que hacen los mejores profesores de universidad. Traducción Óscar Barberá. Valencia: Universidad de Valencia.

Núñez, F. y Arcos, A. (coords.) et al (2018). Factores de éxito de los Estudiantes Universitarios y herramientas para conseguirlo. Colección INNTED. Sevilla: Ediciones Egregius.

Jornadas de Formación e Innovación Docente del Profesorado | № 2 (2019) 